\title{
Fractal growth of epitaxial surface clusters with elastic interaction
}

\author{
J. Steinbrecher, H. Müller-Krumbhaar, E. Brener, C. Misbah, ${ }^{*}$ and P. Peyla ${ }^{\dagger}$ \\ Institut für Festkörperforschung, Forschungszentrum Jülich, D-52425 Jülich, Germany
}

(Received 17 November 1998)

\begin{abstract}
The fractal growth of clusters adsorbed on crystal surfaces has been studied by Monte Carlo simulations. Elastic interactions between the atoms through the substrate have been included. Attractive and repulsive interaction potentials $1 / r^{3}$ have been used, including a varying cutoff for the range of interaction. As an important result we find that there exists a crossover radius beyond which the fractal dimension of the cluster corresponds to the fractal dimension of conventional two-dimensional diffusion limited aggregation. The crossover radius itself and the properties of the cluster inside that radius depend sensitively on the details of the interaction. The results have been analyzed by a scaling theory. Furthermore, we have implemented a multigrid scheme which allows for very efficient simulation of a large number of mobile atoms with long-range interaction on the surface. [S1063-651X(99)09404-0]

PACS number(s): 68.10.Et, 68.55.-a
\end{abstract}

\section{INTRODUCTION}

The formation of monatomic layers of atoms adsorbed on a substrate of different atoms or molecules is a process of great interest in crystal growth [1-3], and for the manufacturing of semiconductor devices. Under low deposition rates clusters are formed on flat substrates. If the surface diffusivity - and in particular the edge diffusivity - is sufficiently low, the clusters will show a ramification during growth. This process has been studied in great detail under the title "diffusion limited aggregation" (DLA) [4].

This so-called epitaxial growth of a new layer on a substrate of different material leads generally to a deformation of the substrate lattice because of a mismatch of the lattice constants of the substrate and the adsorbed material. A single adsorbed cluster up to some limiting size will acquire the lattice structure of the substrate apart from a small local change in the lattice parameter. This "coherent" lattice deformation causes elastic stress in both the adsorbate and the substrate, leading to effective long-range interactions between the adsorbate atoms mediated by the substrate deformations. This effective elastic interaction potential between any two adsorbed atoms is typically repulsive, and depends on their distance $r$ like $1 / r^{3}$ [5-8]. The total interaction energy in an adsorbed cluster is then obtained by the summation of the individual contributions from any two pairs of atoms, as long as the cluster remains coherent with the substrate.

A number of recent investigations have looked at DLA with interactions $[9,10]$. Some investigations even deal with long-range interactions [11-13] of the type discussed here, but we are not aware of any attempts to study the asymptotic behavior of the structures for large cluster sizes. In addition, we have varied the range of interaction systematically by introducing a sharp cutoff which limits the $1 / r^{3}$ potential at

\footnotetext{
*Permanent address: Laboratoire de Spectrometrie, Université J. Fourier, Grenoble, France.

${ }^{\dagger}$ Permanent address: Laboratoire de Physique et de Modélisation des Milieux Condensés, Université J. Fourier, Grenoble, France.
}

some cutoff radius $r_{0}$; furthermore, attractive interactions also were considered for comparison. As a main result we find a change of properties of the growing cluster when the radius exceeds a crossover radius $r_{X}$. This crossover radius depends on the strength and the range of interaction, but remains finite even for the long-range elastic interaction without finite cutoff.

The paper is organized as follows. First we describe the algorithms schematically. This concerns the simple algorithm for DLA with vanishing density of adsorbate atoms far away from the cluster and also the multigrid algorithm for a finite density of atoms at infinity, both algorithms, of course, being effective for long-range potentials. Then we give the numerical results, and finally we present our scaling concept and show that it is consistent with the numerical results within the present numerical accuracy.

\section{ALGORITHMS FOR LONG-RANGE POTENTIALS}

The basis for our cluster-growth algorithm is the original method of Witten and Sander [4,14] for diffusion limited aggregation without interaction, in the version of Meakin [15] for a reduced start radius $r_{s}$. With interaction between particles the probability for starting a particle on the start radius is no longer isotropic over the circle, but depends, through a Boltzmann weight $\exp \left(-U_{s} / T\right)$, upon the local potential on the start circle [12]. The process is now described in some detail.

The substrate is represented as a square lattice of at least $2000^{2}$ sites. One adatom is fixed at the center. Two circles are defined, an outer one with a capture radius $r_{\text {cap }} \approx 1000$ lattice site and an inner one with a start radius $r_{s} \approx 100$ lattice site. The lattice sites carry the potential from the initial central adatom approximately equal to $U_{0} / r^{3}$. A new particle is then released from the start circle, the position chosen in proportion to the Boltzmann weight, as mentioned above. In principle one should choose the probability according to a first passage-time calculation, assuming that the particle starts originally from the outer capture radius. The Boltzmann weight introduces an error which is small when the potential energy close to the cluster is large, and when the 
start radius is far away from the cluster. A small start radius saves computing time. For different values of the prefactor $U_{0}$ of the interaction potential, we have checked that the error introduced by a small start radius, which is very close to the most prominent point of the cluster, is not detectable within our available accuracy. Practically, start radii with distances between four and 100 lattice units from the cluster were used.

After the particle is started, it performs diffusion jumps in a potential field. Several Monte Carlo algorithms were tested; the results presented here were largely obtained by a Metropolis algorithm. This algorithm states [16] that a new trial state is accepted, if either the energy difference $\Delta U$ to the old state is negative or else if $\exp (-\Delta U / T)>X_{r}$, where $X_{r}$ is a random number evenly distributed in the interval between 0 and 1 , and $T$ is the temperature of the system. In each diffusion jump we pick one of four nearest-neighbor sites at random as a trial site, and then make a Metropolis decision for acceptance.

This diffusion process continues until either the particle wanders outside the capture radius, or until it hits the central cluster (one adatom, at first). When it exceeds the capture radius it is removed, and a new particle is inserted at the start radius. When it hits the central cluster at a nearest-neighbor position, it becomes immobilized and is added to the cluster. Then the potential from this particle is added to all lattice sites inside the capture radius. After this a new particle is inserted at the start radius, and so forth. The computing time is not exorbitantly larger than for DLA without interaction, since a full diffusion process on a lattice with diameter $L$ takes $\approx L^{2}$ steps, and so does the updating of the twodimensional lattice after incorporating one atom into the cluster. This process works well as long as there is only one moving particle in the system.

If we have a nonzero density of moving particles with long-range interaction in the system, this direct procedure is too time consuming. We therefore introduced a multigrid algorithm which we now describe in somewhat sketchy form. Most of the following results, however, have been obtained with the simpler algorithm described here above.

The multigrid algorithm starts from the idea that the longrange interaction potential is sufficiently smooth, like a power-law interaction. In this case the relative variation of the interaction, depending upon the distance of the two particles, decreases with the distance. Instead of evaluating the interaction between all pairs of particles explicitly, one can first average over some region of space containing a group of particles, and then take the interaction between one specific particle and the group average only. This, of course, is an approximation which, however, can be systematically improved by subsequent multipole expansions $[17,18]$ of the spatial distribution of the respective group of particles.

The multigrid algorithm employed here groups the lattice sites on every level of a hierarchy into blocks which cover a linear dimension larger by a factor 2 on each successive level. To be explicit let us assume that we have a linear lattice of $L=2^{N}$ sites. We then construct $N$ different grids each one representing a different "level."' Every particle appears on every grid, but the grids on the higher "levels" contain the particles as groups only, on a grid with a larger mesh size. On level 0 we have the original lattice with $2^{N}$
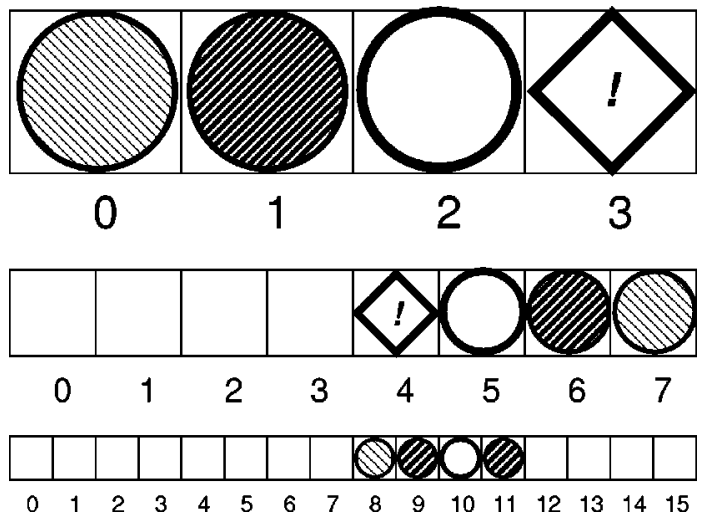

FIG. 1. Schematic structure of successive levels of coarseness in our multigrid method. The reference site on each level is marked by an open circle. The other circles and the diamond mark nearest and next-nearest neighbors on each level, interacting with the reference particle. The diamond corresponds to a box, whose interactions have already been taken into account on the level just below. For further details, see the text.

sites. On level 1 we have performed a coarse graining by a factor of 2 , so that we have $2^{N-1}$ sites. The mesh size accordingly is also increased by a factor 2 . On level 2 another coarse graining takes place, so that we have $2^{N-2}$ sites, and so on. If there is a particle on level 0 at position $i_{0}$, this particle will also appear in the next higher level at position $i_{1}=\operatorname{Int}\left(i_{0} / 2\right)$, where $i_{m}(m=0,1,2,3, \ldots, N)$ are all truncated integers. Note that in principle there may be an arbitrary number of particles at each site $i_{m}(m>0)$. For example, if there are two particles sitting at sites 2 and 3 on level 0 , they both will appear on site 1 on level 1 and on site 0 on level 2 and higher levels.

The interaction between one particle and all other particles in this one-dimensional lattice is now computed as follows. We assume that all particles have been put already in all the $\log _{2}(L)$ levels of our multigrid system. As an example we are discussing now specifically a particle on site $i_{0}=10$, the counting of lattice-sites on each level $m$ starting at $i_{m}=0,1,2, \ldots$ (see Fig. 1).

We first look at the nearest-neighbor interaction of particle 10 , with particle 11 on level 0 . Note that on level 1 particle $i_{0}=10$ would be in site $i_{1}=5$ together with its neighbor $i_{0}=11$, the interaction between those particles has already been treated on level 0 . We now can directly treat the interaction between sites $i_{1}=5$ and $i_{1}=4$ on level 1 , and then proceed to the next higher level. Note that in site $i_{1}$ $=4$ we find particles which were originally on sites $i_{0}=8$ and 9.

This simple process, however, introduces a somewhat strong asymmetry into the treatment of the neighbors $i_{0}$ $=11$ and 9 . We therefore treat also the nearest-neighbor interaction between particles 10 and 9 directly on level 0 , and, even more, also treat the interaction between particles 10 and 8 on level 0 explicitly. The reason for the latter is, that both particles 9 and 8 on level 0 will appear in site $i_{1}=4$ on level 1. If we only take the interaction between particles 10 and 9 on level 0 , leaving out the interaction between particles 10 and 8 on level 0 , obviously we would have a problem on level 1 in the treatment of the interaction between the sites 
$i_{1}=5$ and 4 . Proceeding to the next higher levels, on each level we accordingly consider two nearest-neighbor interactions, one of which has been accounted for already on the level below, and one next-nearest-neighbor interaction.

The generalization to two and higher spatial dimensions is obvious. As soon as one site is included in an interaction on level $m$, all sites have to be included in the same way on this level, if they belong to the same block site on level $m+1$. The other steps of the process are straightforward, and will not be discussed here further. This multigrid process substantially reduces the computing effort for the long-range interactions, since instead of $\sim L^{d}$ interactions, where $d$ is the dimension of the system, we have to consider only $\sim \log _{2}(L)$ interactions. The efficiency decreases, of course, with the decreasing average density of particles in the system.

The approximations introduced here lead to some errors in the results; an overlayer structure, for example, is introduced by this multigrid process. These errors are largest in an intermediate range of interactions. The nearest neighbors are treated exactly, and-for a nonzero average density of particles - the asymptotic contributions for very distant particles are also treated increasingly accurately with increasing distance. The precision of the calculation can be systematically improved at the cost of more operations on each level. For example, instead of only storing the total number of particles in each coarse-graining box and taking the center of the box as their average position, one can explicitly store the average position of the particles in the box and higher moments of their spatial distribution, in order to improve the calculation of relative distances. This is systematically done by a usual multipole expansion $[17,18]$. For our purposes we did not exploit these possibilities since we are more interested in basic qualitative results. For those the correct treatment of short-range interactions and the correct treatment of the long-range tails seemed to be sufficient.

\section{NUMERICAL RESULTS FOR CLUSTERS WITH ELASTIC INTERACTION}

We first present our basic results on the growth of fractal clusters with elastic interaction between particles under a conventional DLA process where only one particle is moving at a time. Afterwards we describe some first results on the propagation of a fractal front growing out of a background of finite density of adsorbed atoms.

The effective elastic interaction $1 / r^{3}$ between a diffusing particle and each atom of an immobilized cluster still leads to the formation of overall fractal clusters, as far as this can be demonstrated by our numerics. When the interaction is repulsive, as is usually the case for elastic forces mediated over the substrate, the resulting cluster looks generally denser than a cluster without long-range repulsive interaction. This is shown in Fig. 2. When the interaction is artificially set to be negative, the cluster looks sparser than without interaction. This was already qualitatively found in previous investigations [12].

The fractal dimension-at least for clusters up to some 40000 particles - seems to depend upon the interaction. This can be seen in Fig. 3, where a log-log plot of the radial density of particles versus the radius is shown. The slope of the fitted curve is equal to $2-D_{f}$, here giving $D_{f} \approx 1.94$ as
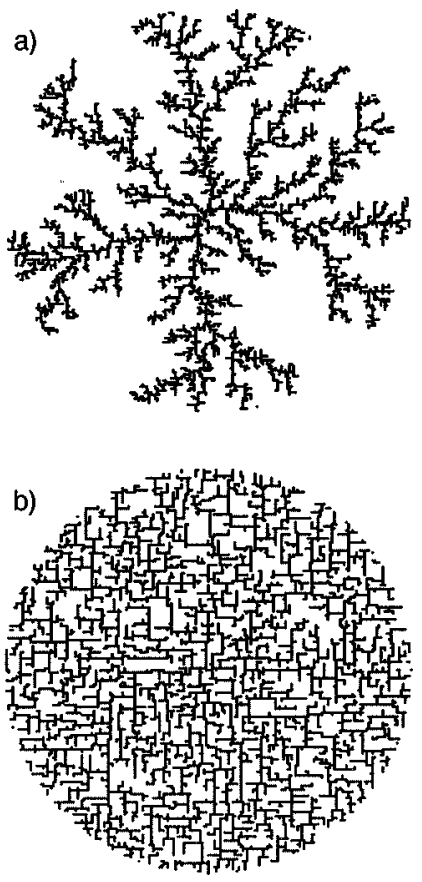

FIG. 2. Comparison of two structures growing via DLA without (a) and with (b) repulsive interaction. Both figures show a central section of 100 lattice units in the radius of a much larger cluster.

the fractal dimension for the cluster grown at a relative potential strength of $U_{0} / T=2.8284$. Note that the absolute potential value is irrelevant, since the particle sticks with infinite strength to the cluster once it has made nearest-neighbor contact with it, and so there is no other energy scale present. In order to see whether these potential effects were already caused by short-range interactions, or whether this is typical of the long-range $1 / r^{3}$ potential, we have introduced a sharp cutoff at varying distance. In Fig. 4 we show an effective fractal dimension evaluated as in Fig. 3 for clusters of up to about $4 \times 10^{4}$ particles. The fractal dimension seems to depend on the strength of the repulsive potential, but not so much on its range. This is a somewhat surprising result, since one would generally not expect a "critical" quantity like the fractal dimension to depend on local forces, as long as they do not change the symmetry of the system.

The influence of our potential cutoff upon the density of the system [to be precise, upon the prefactor $\rho_{0}$ of the radiusdependent density $\rho(r)$ ] seems to be more pronounced, as can be seen in Fig. 5. Again there is a rather strong depen-

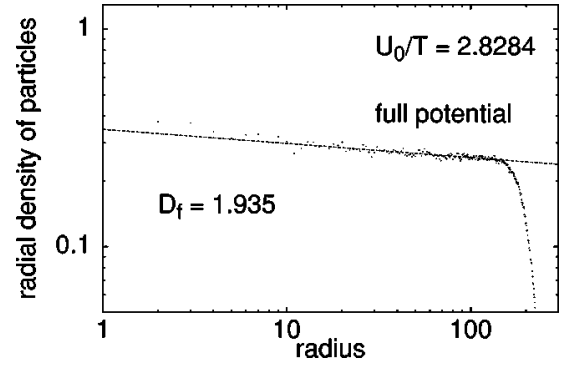

FIG. 3. Radial particle density of a medium-size DLA cluster with repulsive elastic interaction. The effective fractal dimension seems to be close to $D_{f} \approx 2$. 


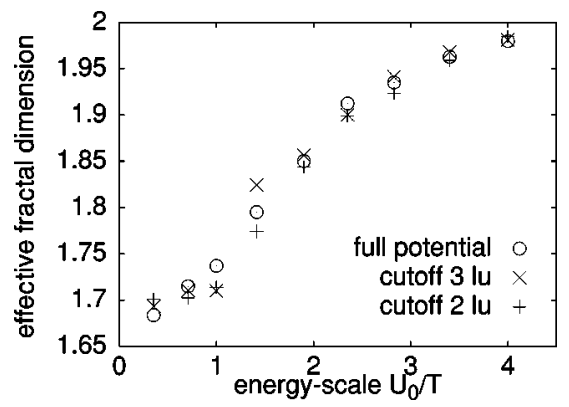

FIG. 4. Effective fractal dimension $D_{f}$ in the central region of the cluster as a function of the repulsive energy $U_{0} / T$ and of the potential cutoff, given in lattice units.

dence upon the strength of the interaction potential, but also the range of the potential now enters more significantly.

For attractive interaction the effect is rather similar, but the change of the fractal dimension goes in the other direction. For a maximal attractive value of $U_{0} / T=-4$ the fractal dimension of clusters up to some 20000 particles changes to the value of $D_{f} \approx 1.55$, which is significantly below the value of about 1.7 for DLA without interaction $[15,19]$.

The overall appearance of the clusters with attractive interaction is much more feathery or thinner than for conventional DLA, while for repulsive interactions the structures look bushier or thicker.

In order to elucidate the dependence of the fractal dimension upon the strength of the interaction, we made some large-scale clusters with relatively strong repulsive potential and with practically an infinite-range cutoff. Such a cluster of some $200000\left(2 \times 10^{5}\right)$ particles is shown in Fig. 6. The arms of the cluster look somewhat "fat," but still indicate conventional fractal behavior. A quantitative analysis is shown in Fig. 7, where, as in Fig. 3, a log-log plot of the radial density depending upon the radius is given. The data represent an average over eight independently grown clusters. What one clearly now sees is an inner region inside a radius of about 140 lattice units, where an effective fractal dimension of $D_{f} \approx 1.85$ can be defined, and an outer region between $140<r<500$ where a DLA value of $D_{f} \approx 1.74$ not significantly different from the conventional value of $\approx 1.71$ is recovered.

These results (Figs. 6 and 7) indicate that even the longrange interaction of $\left(U_{0} / T\right) 1 / r^{3}$ does not change the asymptotic value of the fractal dimension, but only affects

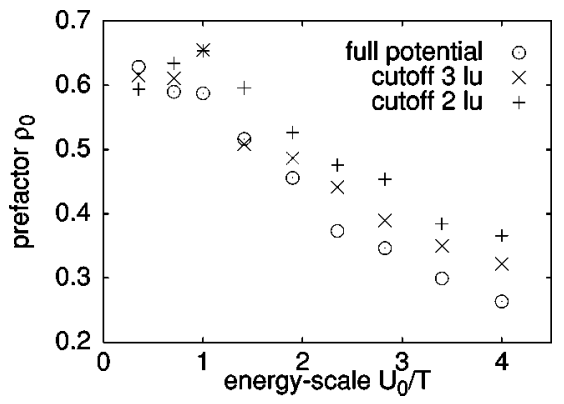

FIG. 5. Prefactor $\rho_{0}$ of the radial density in the central region of the cluster as function of the repulsive energy $U_{0} / T$ and of the potential cutoff.

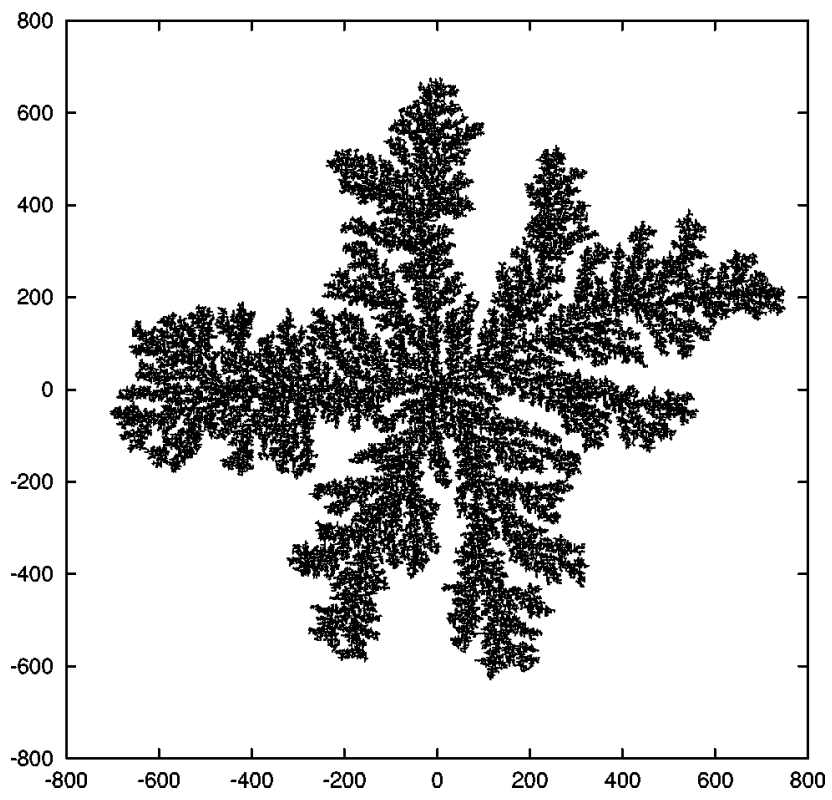

FIG. 6. Cluster of $2 \times 10^{5}$ particles grown by a DLA process. There is a repulsive elastic $1 / r^{3}$ interaction between each two particles, the relative interaction strength being $U_{0} / T=2.0$. The size of the cluster is approximately $1600^{2}$ lattice units.

the center of the cluster and the fine structure of the growing arms of the cluster. This also gives an explanation of why the results with and without cutoff in Figs. 4 and 5 do not differ significantly, since even for infinite cutoff the interaction stays in some sense finite, as will be further discussed in Sec. IV. It is not yet clear, if the different effective fractal dimension observable near the center of the cluster should really be interpreted as a fractal dimension or only as a crossover to a compact cluster of dimension 2 . In our scaling analysis below we will also leave this point open.

A first result of growth structures obtained from the multigrid growth algorithm is shown in Fig. 8. The observed fractal dimensions on length scales shorter than the effective diffusion length (see Ref. [20] for comparison) scales in agreement with Figs. 4 and 5. Note that in contrast to the first intuition, the effective fractal dimension on the right side of Fig. 8 is larger than on the left. Further details will be published elsewhere.

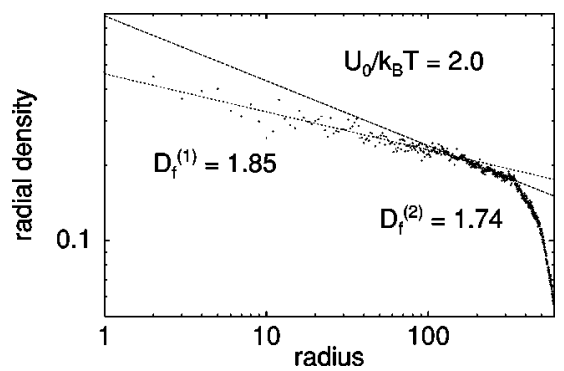

FIG. 7. Log-log plot of radial density vs radius of clusters like the one seen in Fig. 6, with elastic repulsion between particles. The data show an average over eight independently grown clusters. A crossover in the slope of the plot around a radius of $r_{X} \approx 140$, from an effective fractal dimension of $D_{f} \approx 1.85$ near the center to a lower value $D_{f} \approx 1.74$ at large sizes, is clearly seen. 


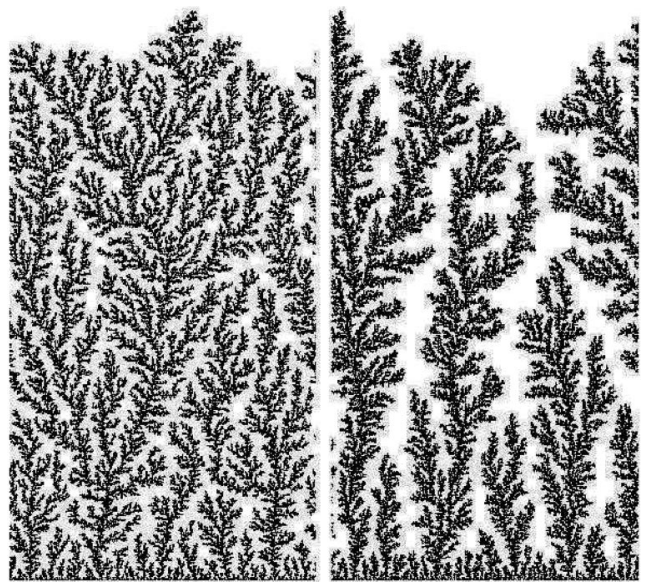

FIG. 8. Growth fronts growing in the upward direction from a two-dimensional gas of nonvanishing density $\rho=0.15$. There is a repulsive $1 / r^{3}$ interaction $U_{0} / T=1.0$ between the atoms in the right figure, and no interaction in the left figure. Note that the effective fractal dimension is lower on the left and higher on the right.

\section{SCALING THEORY FOR CLUSTERS WITH ELASTIC INTERACTION}

The qualitative effect of an interaction between the diffusing particle and a cluster of aggregated particles can be understood as follows. Assume that the interaction is repulsive and decaying with distance and, of course, apart from an infinite attractive force acting to keep the particle fixed at its cluster site, as soon as it has made contact with the cluster.

Assume, for the moment, that a large dense cluster exists of approximately circular shape which has a baylike opening of appreciable depth and a width of several lattice sites at some place on its periphery. A particle near the opening of that bay will "feel" a less pronounced repulsive potential on the axis of that "fjord" or bay, and will with increased probability enter the fjord.

On the axis of the fjord, the repulsive potential relative to the walls of the fjord is a minimum. To be explicit, we assume that the potential barrier to be overcome by the particle in order to move from the axis of the fjord to one of its sides be $\hat{U}$. It is now obvious that the probability of the particle to jump from a position on the axis of the fjord to one of its sides is reduced by a factor of $\sim \exp (-\hat{U} / T)$ compared to a jump of similar distance in direction along the axis of the fjord. In other words, the particle will make on the order of $\exp (+\hat{U} / T)$ more jumps of equal length in a direction along the axis of the fjord than in a direction toward the walls. Consequently, a particle can be expected to travel a distance of $\exp \left(\frac{1}{2} \hat{U} / T\right)$ into such a fjord before it is captured at one of the walls. This explains qualitatively why under repulsive interaction the resulting fractal structures look in some sense denser than without interaction. Furthermore, this explains that a new length scale $r_{X}$ appears in the process,

$$
r_{X} \approx r_{w} \exp \left(\frac{1}{2} \hat{U} / T\right)
$$

which we will interpret just below as a crossover length. The prefactor $r_{w}$ on the right-hand side stands for the width of that fjord. Its minimal value must be larger than about 2 , in order for a particle to enter the fjord without being captured at the entrance. A reasonable value, still of the order of unity but large enough that the potential in the center of the fjord can be neglected, would give $r_{w} \approx 5$ as a rough estimate.

Obviously one can relate this energy scale $\hat{U}$ with the interaction energy $U_{0}$ introduced above. A particle which comes from a distant point to a large dense cluster has to overcome a potential barrier of

$$
\hat{U}=\pi \int_{1}^{r_{0}} d r r U_{0} / r^{3}
$$

which gives simply

$$
\hat{U}=C_{0} U_{0}\left(1-1 / r_{0}\right) .
$$

This representation already contains our artificially introduced cutoff radius $r_{0}$. The constant $C_{0} \approx 3.3$ contains a correction for the discreteness of our square lattice instead of the continuous integral (2).

Inserting numbers, one quickly finds that the crossover radius of $r_{X} \approx 140$ observed in Fig. 7 is also within the same order of magnitude as obtained from the scaling results [Eqs. (1) and (3)]. Unfortunately it is not easy to study the variation of the crossover radius with the interaction strength numerically with satisfactory precision: if the relative potential $U_{0} / T$ is significantly smaller than the value of 2 , the effective dimension near the center is not significantly different from the value $\approx 1.7$ of the asymptotics, and the crossover point cannot be located without ambiguity. If the interaction potential is much stronger, the repulsion leads to an enormous slowing down of the aggregation process, and the statistics over available computing times become very poor.

Our central argument, therefore, comes from the results for the numerical parameters given in Figs. 6 and 7, which are in good agreement with the scaling formulas. A few corrections could have been included into these formulas-for example, that, of course, the aggregating cluster is not really dense-but this would have been too much analytical detail in comparison to numerical evidence. In any case the data give strong support for the concept that even for this longrange potential of $1 / r^{3}$ the asymptotic scaling of the growing fractal cluster is not changed from the behavior observed without additional interaction.

In summary we have studied the growth of twodimensional fractal adsorbate clusters growing by a DLA process with effective long-range elastic $1 / r^{3}$ interaction between the adsorbate atoms. We find, as a central result, that there exists a crossover radius $r_{X}$ (depending exponentially upon the strength of the interaction), outside of which the cluster grows with the same fractal dimension of about $D_{f}$ $\approx 1.7$ as observed in conventional DLA. Inside that crossover radius the fractal dimension of the cluster torso seems to be increasing toward a value of $D_{f}=2$ for increasing repulsive interaction, and decreasing smoothly toward $D_{f} \approx 1.5$ for an increasingly attractive $1 / r^{3}$ interaction. One can finally speculate that the fractal dimension would change even asymptotically if the interaction potential were to decay only with $1 / r^{2}$, as was recently found for the Eden model [21]; however, this is beyond the scope of the present investiga- 
tions. Our results indicate that for practical applications, e.g., in molecular-beam epitaxy, the cluster properties will depend in a rather sensitive way upon the lattice mismatch between the adsorbate and substrate since this directly affects the interaction parameter which enters exponentially into the crossover radius.

\section{ACKNOWLEDGMENTS}

We thank V. Marchenko, Y. Saito, and D. Temkin for discussions. This work was supported by a grant from the Volkswagen Foundation and by a European Procope travel grant.
[1] Y. Saito, Statistical Physics of Crystal Growth (World Scientific, Singapore, 1996).

[2] R. Nötzel, J. Temmyo, and T. Tamamura, Nature (London) 369, 131 (1994).

[3] R. Jullien, J. Kertesz, P. Meakin, and D. E. Wolf, Surface Disordering: Growth, Roughening and Phase Transitions (Nova, Commack, NY, 1993).

[4] T. A. Witten and L. M. Sander, Phys. Rev. Lett. 47, 1400 (1981).

[5] J. Hardy and R. Bullough, Philos. Mag. 15, 237 (1967).

[6] K. H. Lau and W. Kohn, Surf. Sci. 65, 607 (1977).

[7] V. I. Marchenko and A. Y. Parshin, Zh. Éksp. Teor. Fiz. 79, 257 (1980) [Sov. Phys. JETP 52, 129 (1980)].

[8] A. F. Andreev and Y. A. Kosevich, Zh. Éksp. Teor. Fiz. 81, 1435 (1981) [Sov. Phys. JETP 54, 761 (1981)].

[9] N. Vandewalle and M. Ausloos, Phys. Rev. E 51, 597 (1995).

[10] Rong-Fu Xiao, J. I. D. Alexander, and F. Rosenberger, Mater. Sci. Eng., A 178, 233 (1994).
[11] R. Pastor-Satorras and J. M. Rubí, Phys. Rev. E 51, 5994 (1995).

[12] G. Indiveri, A. C. Levi, A. Gliozzi, E. Scalas, and H. Möhwald, Thin Solid Films 284, 106 (1996).

[13] P. Peyla, A. Vallat, C. Misbah, and H. Müller-Krumbhaar, Phys. Rev. Lett. 82, 787 (1999).

[14] T. A. Witten and L. M. Sander, Phys. Rev. B 27, 5686 (1983).

[15] P. Meakin, Phys. Rev. A 27, 1495 (1983); 32, 453 (1985).

[16] The Monte Carlo Method in Statistical Physics, edited by K. Binder (Springer-Verlag, Berlin, 1977).

[17] J. T. Hamilton and G. Majda, J. Comput. Phys. 121, 29 (1995); C. L. Berman, SIAM J. Comput. 16, 1082 (1995).

[18] V. Jandhyala, E. Michielssen, and R. Mittra, Int. J. Microwave Millimeter-Wave Computer Aided Eng. 6, 381 (1996).

[19] P. Ossadnik, Phys. Rev. A 45, 1058 (1992).

[20] M. Uwaha and Y. Saito, Phys. Rev. A 40, 4716 (1989).

[21] Y. Saito and H. Müller-Krumbhaar, J. Phys. Soc. Jpn. 67, 3661 (1998). 